\title{
AN AMENABLE GROUP WITH A NONSYMMETRIC GROUP ALGEBRA ${ }^{1}$
}

\author{
BY JOE W. JENKINS
}

\author{
Communicated by Paul T. Bateman, November 4, 1968
}

Let $G$ be a discrete group, $l_{1}(G)$ the group algebra of $G$. Symmetry of $l_{1}(G)$ has been considered in [1], [3]. Groups containing a free subgroup on two or more generators are the only groups found to have nonsymmetric group algebras, and in each case the groups found to have symmetric algebras are in the family of amenable groups. In this note we present an example of an amenable group with a nonsymmetric group algebra.

Leмma 1. Let $G$ be a group generated by $a$ and $b$ such that $S$, the semigroup generated by $a$ and $b$, is free and such that $c d^{-1}=d c^{-1}$ for $\{c, d\}$ $=\{a, b\}$. Then $l_{1}(G)$ is nonsymmetric.

Proof. We will show that $l_{1}(G)$ is not symmetric by showing that the involution is not hermitian. In particular, we will show that $-i \in \operatorname{sp}(x)$ where

$$
x=a+i b+a^{-1}-i b^{-1}
$$

(we do not distinguish between $G$ and its canonical image in $l_{1}(G)$ ). This is accomplished by defining a $\theta$ in $m(G)$, the bounded complex valued functions on $G$, such that $\|\theta\|=1=\theta(e)$ and such that

$$
\theta^{\circ}[(x+i e) g]=0
$$

for each $g \in G$, where $\theta \rightarrow \theta^{v}$ is the mapping of $m(G)$ onto $l_{1}(G)^{*}$.

Let $S^{\prime}=S \cup S^{-1} \cup\{e\}$, and define $\theta(g)=0$ for $g \in G \sim S^{\prime}$.

We divide the elements of $G$ into the five disjoint sets; $S, \quad S^{-1}, \quad S_{1}=a^{-1} b S \cup\left\{a^{-1} b\right\}, \quad S_{2}=a b^{-1} S^{-1} \cup\left\{a b^{-1}\right\}$ and $S_{3}$ $=G \sim\left(S \cup S^{-1} \cup S_{1} \cup S_{2}\right)$. Let $A=\left\{a, b, e, a^{-1}, b^{-1}\right\}$. Direct computations yield $A g \cap S^{\prime} \neq \varnothing$ if and only if $g \notin S_{3}$ or $g=e$.

Note now that if $g \in S_{3}$ and $g \neq e$ then

$$
\begin{aligned}
{[\operatorname{support}(x+i e) g] \cap S^{\prime} } & =A g \cap S^{\prime} \\
& =\varnothing,
\end{aligned}
$$

1 The results announced here are contained in the author's doctoral dissertation written at the University of Illinois under the guidance of Professor M. M. Day. Partial support was received from National Science Foundation Grant GP-6990. 
and hence,

$$
\begin{aligned}
\theta^{0}[(x+i e) g] & =\sum_{t \in G}[(x+i e) g](t) \theta(t) \\
& =0 .
\end{aligned}
$$

We enumerate the elements of $S^{\prime}$ as follows:

$$
\begin{aligned}
s_{0} & =e ; \quad \text { for } n=0,1,2, \cdots, s_{2 n+1}=a s_{n}, \\
s_{2 n+2} & =b s_{n}, s_{-(2 n+1)}=a^{-1} s_{-n}, \\
s_{-(2 n+2)} & =b^{-1} s_{-n} ; \quad \text { for } n=1,2,3, \cdots, \\
t_{n} & =a^{-1} s_{2 n}, t_{-n}=a s_{-2 n} .
\end{aligned}
$$

Let $y=x+i e$. We have

$$
\begin{aligned}
\theta^{v}(y) & =\theta\left(s_{1}\right)+i \theta\left(s_{2}\right)+\theta\left(s_{-1}\right)-i \theta\left(s_{-2}\right)+i \theta\left(s_{0}\right), \\
\theta^{0}\left(y t_{1}\right) & =i \theta\left(s_{1}\right)+\theta\left(s_{2}\right), \\
\theta^{v}\left(y t_{-1}\right) & =\theta\left(s_{-2}\right)-i \theta\left(s_{-1}\right) .
\end{aligned}
$$

$\operatorname{Set} \theta\left(s_{0}\right)=\theta(e)=1$. The following system of equations has a solution such that $0<\left|\theta\left(s_{i}\right)\right| \leqq 1$ for $-2 \leqq i \leqq 2$;

$$
\begin{aligned}
\theta\left(s_{1}\right)+i \theta\left(s_{2}\right)+\theta\left(s_{-1}\right)-i \theta\left(s_{-2}\right) & =-i, \\
i \theta\left(s_{1}\right)+\theta\left(s_{2}\right) & =0, \\
-i \theta\left(s_{-1}\right)+\theta\left(s_{-2}\right) & =0 .
\end{aligned}
$$

With these values of $\theta\left(s_{i}\right),-2 \leqq i \leqq 2$, we have $\theta^{v}(y)=0, \theta^{v}\left(y t_{1}\right)=0$, and $\theta^{v}\left(y t_{-1}\right)=0$.

Now

$$
\theta^{v}\left(y s_{1}\right)=1+i \theta\left(s_{1}\right)+\theta\left(s_{2}\right)+i \theta\left(s_{4}\right)
$$

and

$$
\theta^{v}\left(y t_{2}\right)=i \theta\left(s_{3}\right)+\theta\left(s_{4}\right)
$$

The equations

$$
\begin{aligned}
& \theta\left(s_{3}\right)+i \theta\left(s_{4}\right)=-1-i \theta\left(s_{1}\right), \\
& i \theta\left(s_{3}\right)+\theta\left(s_{4}\right)=0,
\end{aligned}
$$

have a solution such that $0<\left|\theta\left(s_{i}\right)\right| \leqq 1$ for $i=3,4$. Thus, with these values for $\theta\left(s_{3}\right)$ and $\theta\left(s_{4}\right)$, we have $\theta^{v}\left(y s_{1}\right)=0$ and $\theta^{v}\left(y t_{2}\right)=0$.

For $k$ an arbitrary positive integer,

$$
\theta^{v}\left(y s_{k}\right)=\theta\left(s_{2 k+1}\right)+i \theta\left(s_{2 k+2}\right)+i \theta\left(s_{k}\right)+(-1)^{k-1} \theta\left(s_{k^{\prime}}\right)
$$


and

$$
\theta^{0}\left(y t_{k+1}\right)=i \theta\left(s_{2 k+1}\right)+\theta\left(s_{2 k+2}\right),
$$

where $k^{\prime}$ is the largest integer less than or equal to $(k-1) / 2$.

Hence, assuming $\theta\left(s_{j}\right)$ has been assigned for $0 \leqq j \leqq 2 k$ and that $0<\left|\theta\left(s_{j}\right)\right| \leqq 1$, the following equations have a solution such that $0<\left|\theta_{2 k+1}\right| \leqq 1$ and $0<\left|\theta_{2 k+2}\right| \leqq 1$;

$$
\begin{aligned}
\theta\left(s_{2 k+1}\right)+i \theta\left(s_{2 k+2}\right) & =-i \theta\left(s_{k}\right)+(-1)^{k-1} \theta\left(s_{k^{\prime}}\right), \\
i \theta\left(s_{2 k+1}\right)+\theta\left(s_{2 k+2}\right) & =0 .
\end{aligned}
$$

Therefore, we can define $\theta(s)$ for each $s \in S$ such that $\theta^{v}\left(y s^{\prime}\right)=0$ for each $s^{\prime} \in S \cup S_{1}$. Similarly, we can define $\theta(s)$ for each $s \in S^{-1}$ so that $\theta^{v}\left(y s^{\prime}\right)=0$ for each $s^{\prime} \in S^{-1} \cup S_{2}$. Hence, $\theta^{v}(y s)=0$ for each $s \in G$.

In [2] an example is given of an amenable group that contains a free subsemigroup on two generators. We will construct a similar example in which the generators of the free subsemigroup satisfy a certain relation within the group.

Let $L(R)$ be the group of ordered pairs of real numbers $(u, v), u \neq 0$, with multiplication defined by the formula

$$
(u, v)\left(u_{1}, v_{1}\right)=\left(u u_{1}, u v_{1}+v\right) \text {. }
$$

ThEOREM 2. Let $s=(a, 1)$ and $t=(-a, 1), 0<a \leqq 1 / 2$, be elements of $L(R)$. Let $G_{0}$ be the group generated by $s$ and $t$. Then $G_{0}$ is amenable and $l_{1}\left(G_{0}\right)$ is nonsymmetric.

Proof. $L_{1}=\{(1, b) \mid b \in R\}$, the commutator subgroup of $L(R)$, is Abelian. Thus, $G_{0} \cap L_{1}$ is Abelian. Also

$$
G_{0} / G_{0} \cap L_{1} \cong G_{0} L_{1} / L_{1} \subset L(R) / L_{1},
$$

and hence $G_{0} / G_{0} \cap L_{1}$ is Abelian. Therefore $G_{0}$, the extension of an Abelian group by an Abelian group, is amenable.

To show that $l_{1}\left(G_{0}\right)$ is nonsymmetric, we first note that $s t^{-1}$ $=(-1,2)=t s^{-1}$. Hence $c d^{-1}=d c^{-1}$ if $\{c, d\}=\{s, t\}$.

An induction argument shows that each word $w$ in $S$ is of the form

$$
w=\left((-1)^{\bullet} a^{n}, \sum_{i=0}^{n-1} p(i) a^{i}\right)
$$

for some positive integer $n$, where $\epsilon=0,1$ and $p(i)= \pm 1$ for $0 \leqq i$ $\leqq n-1$.

If for two distinct words $w_{1}$ and $w_{2}$ in $S$ we have $w_{1}=w_{2}$, then, by 
cancelling like factors on the left, we may assume we have distinct words $w_{3}$ and $w_{4}$ such that $s w_{3}=t w_{4}$. But the second term of $s w_{3}$ is

$$
1+a+\sum_{i=2}^{n} p(i) a^{i}>1
$$

with $p(i)= \pm 1$ and some integer $n$, and the second term of $t w_{4}$ is

$$
1-a+\sum_{j=2}^{m} q(j) a^{j}<1
$$

with $q(j)= \pm 1,2 \leqq j \leqq m$ and some integer $m$. Hence $w_{1} \neq w_{2}$ for distinct words $w_{1}$ and $w_{2}$ in $S$. Therefore, the semigroup generated by $s$ and $t$ is free.

Lemma 1 now applies.

\section{REFERENCES}

1. R. A. Bonic, Symmetry in group algebras of discrete groups, Pacific J. Math. 11 (1961), 73-94.

2. M. Hochster, Subsemigroups of amenable groups (to appear).

3. A. Hulanicki, On the spectral radius of hermitian elements in group algebras, Pacific J. Math. 18 (1966), 277-290.

State University of New York at Albany, Albany, New York 12203 